\title{
Millimeter-wave center of curvature test for a fast paraboloid
}

\author{
Samuel Goldberg and Stephen Padin* \\ Caltech MC367-17, Pasadena, California 91125, USA \\ *Corresponding author: spadin@caltech.edu \\ Received 5 July 2011; accepted 11 September 2011; \\ posted 4 October 2011 (Doc. ID 150429); published 16 January 2012
}

\begin{abstract}
We describe a technique for measuring the surface profile of a radio telescope with a fast paraboloidal primary. The technique uses a sensor, at the center of curvature of the primary, consisting of a millimeterwave source and an array of receivers to measure the field in the caustic. The sensor is mounted on the telescope enclosure and it moves with the telescope, so the measurements can be used for continuous, slow, closed-loop control of the surface. Sensor decenter and despace errors, due to wind buffeting and thermal deformation of the sensor support, do not compromise the surface measurements because they result in profile errors that are mainly translation, which has no effect on astronomical observations, or tilt and defocus, which can be measured using astronomical sources. If the position of the sensor is known to $20 \mu \mathrm{m} \mathrm{rms}$, the surface can be measured to $\sim 1 \mu \mathrm{m} \mathrm{rms}$ at $\lambda=3 \mathrm{~mm}$. (C) 2012 Optical Society of America

OCIS codes: $\quad 120.6650,090.2910,110.6770,350.1260$.
\end{abstract}

\section{Introduction}

This paper describes a technique for measuring the surface profile of a radio telescope. The work was motivated by the Cerro Chajnantor Atacama telescope (CCAT), which will be a $25 \mathrm{~m}$ diameter, submillimeter-wave telescope on a mountaintop in the Atacama Desert [1]. CCAT will be a survey machine, equipped with wide-field cameras and multiobject spectrometers operating in the $0.2-2 \mathrm{~mm}$ wavelength range [2]. The primary surface will be made of $\sim 2 \times 2 \mathrm{~m}$ segments, each with $\sim 16$ reflecting tiles mounted on a rigid subframe. The segments will be supported by a stable, carbon-fiber-reinforced-plastic (CFRP) truss [3]. The primary will be large enough to require active control to compensate gravitational and thermal deformations, so each segment will have three computer-controlled actuators.

Measurements of the surface profile of the primary will be needed to control the surface. Changes in the surface can be measured using edge sensors [4] or

1559-128X/11/030316-07\$15.00/0

(C) 2012 Optical Society of America ranging instruments $[5,6]$, but these must be calibrated based on an absolute measurement of the surface. Such sensors also measure specific points, e.g., the locations of retro reflectors, so local thermal deformations are a problem. A measurement of the average surface error on the scale of a segment or tile is more useful. Low-order surface errors can easily be measured using through focus holography on an astronomical source $[7,8]$. High-order errors can be measured using a shearing or point diffraction interferometer [9-12], but the observations require a bright source. The only astronomical sources that are bright enough are planets. A measurement of the surface with $0.5 \mathrm{~m}$ spatial resolution will take just a few minutes on Mars at $\lambda=350 \mu \mathrm{m}$, but hours on the outer planets [13]. Unfortunately, Mars is only available $\sim 30 \%$ of the time and it will often be so close to Earth that it will be resolved.

What is really needed is a bright artificial source that moves with the telescope, so the surface can be measured quickly at any time. A comoving artificial source cannot be mounted far from the telescope and it cannot be closer than the center of curvature $(\mathrm{CoC})$ of the primary. The latter configuration is used in the 
Hobby Eberly telescope, which has a spherical primary that can image an optical source on a tower at the $\mathrm{CoC}$ [14]. CCAT will be a Ritchey-Chretien design for wide field of view and it will have a short primary focal length to minimize the size and cost of the enclosure. Its fast hyperboloidal primary will produce a poor image of a source at the $\mathrm{CoC}$, so it is not obvious that a $\mathrm{CoC}$ test is viable. In this paper, we demonstrate that the surface profile of the primary can be recovered from measurements of the field in the caustic. This is an extension of the holography technique that is often used to measure the surface profile of radio telescopes $[15,16]$ and mirrors [17]. The CCAT primary will be close to a paraboloid, so the analysis presented here is for a paraboloidal mirror. The material is organized as follows. In Section 2 we describe the test technique, mounting of the $\mathrm{CoC}^{-}$sensor, and the number and position of receivers. In Section 3 we discuss sensor alignment errors. In Section $\underline{4}$ we $\bar{d}$ escribe the effects of obscuration by the secondary.

\section{Center of Curvature Test for a Paraboloid}

The basic configuration of the $\mathrm{CoC}$ test is shown in Fig. 1 . The sensor has a millimeter-wave source, with a feedhorn designed to illuminate the primary with a spherical wave. The source is surrounded by an array of receivers that sample the field over the caustic. Each receiver has a feedhorn-coupled mixer feeding a phase-sensitive detector referenced to the source. The sensor is mounted just beyond the center of curvature, to minimize the diameter of the caustic at the receiver array. For the CCAT primary, which has a focal length of $10 \mathrm{~m}$, the optimum sensor location is $22.7 \mathrm{~m}$ from the vertex of the primary. This gives a $2.4 \mathrm{~m}$ diameter caustic, which fits in the shadow of the $3 \mathrm{~m}$ diameter secondary mirror. All the sensor components are inexpensive, commerciallyavailable devices, such as those sold for automotive radar applications.

The CoC sensor cannot be mounted directly on the telescope without introducing severe structural deformations, so in this case it is mounted on a lightweight, CFRP, low-obscuration tripod attached to

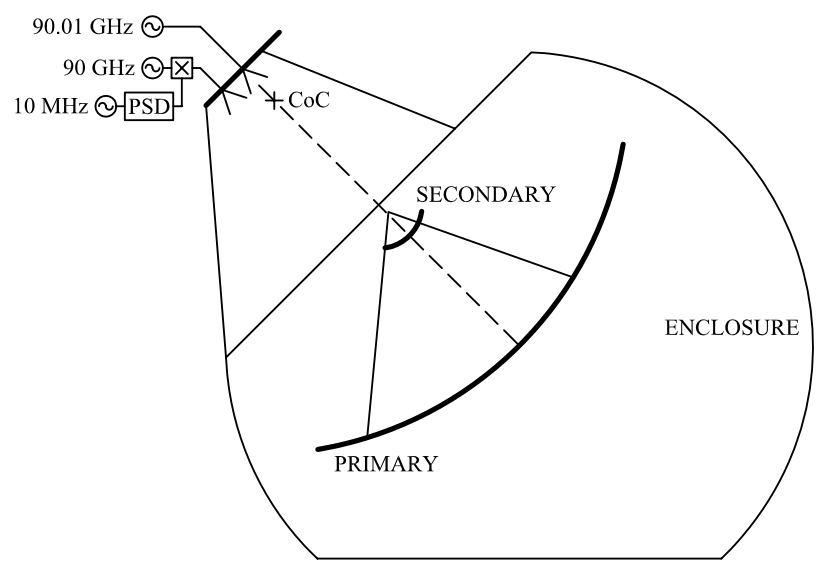

Fig. 1. CoC test. PSD is a phase-sensitive detector. $\times$ is a mixer. the enclosure. The sensor moves with the telescope, so it is always available for slow, closed-loop control of the surface. Mounting the sensor on the enclosure means we have to deal with alignment errors due to wind buffeting, thermal deformation of the sensor support, and different centers of rotation for the enclosure and telescope. To first order, a decenter or tilt of the sensor is equivalent to a pointing error and decenter of the primary, and a despace of the sensor is equivalent to a despace and defocus of the primary. Translation of the primary has no effect on observations, and pointing and focus can be measured quickly using astronomical sources, so these terms can be ignored in the $\mathrm{CoC}$ test, leaving just the higher-order modes which are not easily obtained from astronomical measurements. Removing the translation, pointing, and focus terms allows us to use a light, thin sensor support, but a single measurement of the surface must be made quickly enough to freeze any motion of the sensor. Measurements can then be stacked to improve the signal-to-noise ratio.

First, we demonstrate that the measurements can be made quickly enough. A sensor decenter $\delta$ corresponds roughly to a tilt $\delta$ across the primary. For CCAT, we need to know the surface to a few micrometers rms, so the sensor must move no more than a few micrometers during a measurement. Wind buffeting on a lightweight structure the size of CCAT might cause sensor motion $\sim 10 \mathrm{~mm} \mathrm{~s}^{-1}$, in which case the measurement time must be $\sim 100 \mu \mathrm{s}$. If we measure $n$ patches on the primary surface, the signal-to-noise ratio at a receiver due to one patch is

$$
\mathrm{SNR} \sim(P / n)\left(A / D_{c}^{2}\right) /\left(k_{B} T_{\mathrm{rx}} b\right),
$$

where $P$ is the source power, $A$ is the area of a receiver feedhorn, $D_{c}$ is the diameter of the caustic, $k_{B}$ is Boltzmann's constant, $T_{\text {rx }}$ is the single sideband receiver noise temperature, and $b$ is the predetection bandwidth. The first term in Eq. (1) is the power incident on a single patch on the surface. The second term is the filling factor of the receiver in the caustic. The third term is the receiver noise. We must measure three patches per segment to obtain the tip, tilt, and piston error, so $n \sim 500$ for CCAT. The source and receiver feedhorns must have fairly uniform response over the entire primary. The CCAT primary subtends an angle of $\sim 1 \mathrm{rad}$ at the CoC, so the feedhorn diameter must be $\sim \lambda$ and $A \sim \lambda^{2}$. The phase noise after phase detection is $[\underline{18}, \underline{19}]$

$$
\phi=(\mathrm{SNR} \times b \tau)^{-1 / 2},
$$

where $\tau$ is the measurement integration time. $n$ receivers are required to measure $n$ patches on the primary, so the phase noise is improved by a factor $\sqrt{n}$ and the error in the surface profile is

$$
\sigma=\frac{\lambda}{2 \pi} \phi \sim\left(\frac{D_{c}}{2 \pi}\right)\left(\frac{k_{B} T_{\mathrm{rx}}}{P \tau}\right)^{1 / 2} .
$$


Equation (3) is independent of $n$ because as more patches are measured, more receivers must be added to the sensor. It is also independent of $\lambda$ because the receiver collecting area scales with $\lambda^{2}$. For CCAT, $D_{c} \approx 2.4 \mathrm{~m}$. With $T_{\mathrm{rx}}=10^{4} \mathrm{~K}$ (i.e., an inexpensive, $\lambda=3 \mathrm{~mm}$, room-temperature mixer), and $\tau=$ $100 \mu \mathrm{s}, \sigma<1 \mu \mathrm{m} \mathrm{rms}$ requires $P>1 / 4 \mathrm{~mW}$, which is quite practical.

We now discuss the details of the $\mathrm{CoC}$ measurement. We assume a linearly polarized source with a scalar feedhorn, and we represent the field at the primary as [20]

$$
\begin{aligned}
E_{\theta} & =\left(\frac{P}{\pi}\right)^{1 / 2} \frac{1}{r_{1}} \exp \left(i k r_{1}\right) \sin E_{\phi} \\
& =\left(\frac{P}{\pi}\right)^{1 / 2} \frac{1}{r_{1}} \exp \left(i k r_{1}\right) \cos \phi \mathbf{B}=\nabla \times \mathbf{E} / i \omega,
\end{aligned}
$$

where $\theta$ and $\phi$ are the polar and azimuthal angles, the field is polarized along the direction $\phi=\pi / 2$, $k=2 \pi / \lambda, r_{1}$ is the distance from the source to a point on the primary, $\omega$ is the angular frequency, and the $(P / \pi)^{1 / 2}$ scale factor is for a feedhorn that radiates into just one hemisphere. In our simulations, Eq. (4) gives $\nabla \cdot \mathbf{E}<k \mathbf{E} / 10^{4}$ and $\nabla \times \mathbf{B}<$ $(-i k / c) \overline{\mathbf{E}} / 10^{4}$, so our field model satisfies Maxwell's equations to one part in $10^{4}$. This corresponds to a wavefront error of $10^{-4} / k$, i.e., $\sim 0.02 \mu \mathrm{m}$ surface error at $\lambda=3 \mathrm{~mm}$, which is negligible for a submillimeter-wave telescope. The electric field at receiver $i$ due to radiation from patch $j$ on a perfectly reflecting primary is [21]

$$
\mathbf{E}_{i j}=\int_{j}[(\hat{\mathbf{n}} \times \mathbf{E}) \times \nabla G+(\hat{\mathbf{n}} \cdot \mathbf{E}) \nabla G+i \omega(\hat{\mathbf{n}} \times \mathbf{B}) G] d A,
$$

where $\mathbf{E}$ and $\mathbf{B}$ are the reflected field at the primary, $\hat{\mathbf{n}}$ is the unit normal to the primary, $d A$ is an element of area on patch $j$, and

$$
G\left(r_{2}\right)=\frac{1}{4 \pi r_{2}} \exp \left(i k r_{2}\right)
$$

is the Green's function, where $r_{2}$ is the distance from receiver $i$ to a point on patch $j$. The field at receiver $i$ due to all the patches is then

$$
E_{i}=\sum_{j} E_{i j} v_{j}
$$

where

$$
v_{j}=\exp \left(2 i k x_{j}\right)
$$

is a phase factor that accounts for piston error $x_{j}$ at point $j$ on the primary and $E_{i j}$ is the $\phi=\pi / 2$ component of $\mathbf{E}_{i j}$, selected by the waveguide at the receiver input. Equation (7) can be solved using the least squares method to determine piston errors on the surface.

To simulate a $\mathrm{CoC}$ measurement, we divide the $25 \mathrm{~m}$ diameter primary into 10 rings, each with 10 sectors, i.e., 100 patches in total. A real CoC sensor for CCAT will measure $\sim 500$ patches, but 100 is convenient for a demonstration. First, we precompute the matrix of field contributions, $E_{i j}$, corresponding to a given sensor geometry. We assume initially that the sensor is not buffeted by the wind, so its location is known. We then generate a vector of random surface errors $x_{j}$, and the corresponding phase factors, $v_{j}$. To each $E_{i}$, we add receiver noise

$$
N_{i}=\left(\frac{2 Z_{0} k_{B} T_{\mathrm{rx}}}{A \tau}\right)^{1 / 2} N(0,1) \exp [i \psi(0,2 \pi)]
$$

where $Z_{0}$ is the impedance of free space, $N(0,1)$ is a random variable in the range 0 to 1 , and $\psi(0,2 \pi)$ is a random variable in the range 0 to $2 \pi$. $N_{i}$ is the rms field corresponding to noise power $k_{B} T_{\text {rx }} / \tau$ at the feedhorn, i.e., rms noise voltage $\left(k_{B} T_{\mathrm{rx}} / \tau\right)^{1 / 2}$ at the phase-sensitive detector output. The measured surface profile is given by

$$
v_{j}^{\prime}=\exp \left(2 i k x_{j}^{\prime}\right)=E_{i j}^{-1}\left(E_{i}+N_{i}\right),
$$

where $E_{i j}^{-1}$ is the pseudoinverse of $E_{i j}$. The measurement error is

$$
e=\left\langle\left(x_{j}^{\prime}-x_{j}\right)^{2}\right\rangle^{1 / 2}
$$

For all the simulations in this paper, $\lambda=3 \mathrm{~mm}$, $T_{\mathrm{rx}}=10^{4} \mathrm{~K}, P=5 \mathrm{~mW}$, and $\tau=100 \mu \mathrm{s}$. The calculation of $E_{i j}^{-1}$ takes about five minutes on an Intel Core i5-750 processor for 100 receivers and an integration step size that gives $\sim 0.02 \mu \mathrm{m}$ rms surface error.

Figure 2 shows the field intensity in the caustic. The field has a complicated structure, so the strategy for placing receivers is not obvious. A receiver in the caustic sees radiation from the whole primary, but rings on the primary tend to reflect radiation to rings on the receiver array. Rings 1,8 , and 9 on the primary focus radiation near the center of the receiver array, while rings 3-6 focus radiation around the edge. We can determine how to optimally place a given number of receivers by systematically comparing the measurement error due to different receiver configurations. With 100 patches on the primary, at least 100 receivers are needed to uniquely determine the $v_{j}^{\prime}$. Figure $\underline{3}$ shows the optimum placement for 100 receivers, with positions chosen to minimize the average of the rms error from several measurements (each with a different instance of the receiver noise vector $\mathbf{N}$ ). As expected, the optimum placement gives roughly uniform sampling of the caustic. With 100 optimally placed receivers, the measurement error is $0.7 \mu \mathrm{m} \mathrm{rms}$ (see Fig. 4). If the number of receivers is $n>150$, the measurement error scales roughly as 

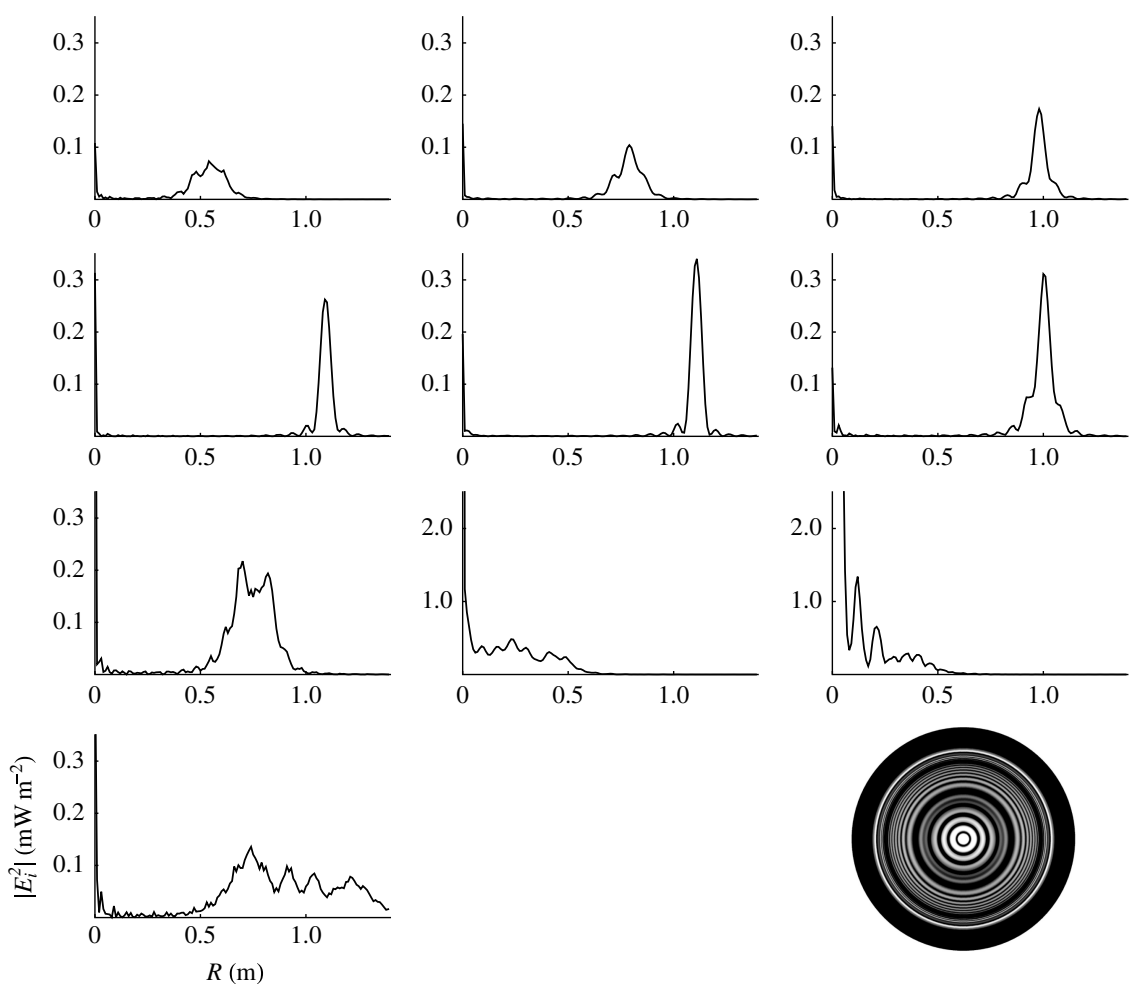

Fig. 2. Field intensity versus radius in the caustic for primary rings 1 (top left) through 3 (top right) through 10 (bottom left) and the entire primary (bottom right, gray scale linear in intensity). Ring 1 is at the center of the primary. Ring 10 is at the edge. Note the change in scale for rings 8 and 9 .

$(n-100)^{-1 / 2}$. It is probably impractical to have many more receivers than patches on the primary.

We have found that configurations in which the receivers are allowed to lie anywhere on the sensor (as in Fig. 3) generally lead to high sensitivity to sensor position errors. Configurations which match the circular symmetry of the field in the caustic (as in Fig. $\underline{5}$ ) are better.

Figure 6 shows a simulation of a $\mathrm{CoC}$ measurement of the CCAT primary with 120 receivers optimally placed in a circularly symmetric configuration. The measurement error is $0.2 \mu \mathrm{m} \mathrm{rms}$ and is un-

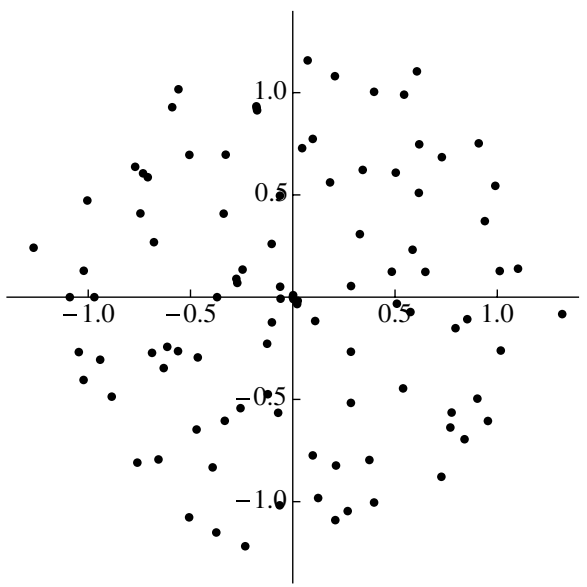

Fig. 3. Optimum placement for 100 receivers. Receiver positions are in meters. correlated with the surface deformations, so it is due to receiver noise rather than systematic errors in the technique.

\section{Alignment Errors}

In Section 2 , we showed that the surface profile of the primary can be recovered if the position of the $\mathrm{CoC}$ sensor is known. In practice, the sensor will move due to thermal deformation of its support, wind buffeting, and different centers of rotation for the telescope and enclosure. In this section, we investigate the effect of sensor position errors.

We first calculate $E_{i}$ with the sensor displaced, then we calculate the surface profile using $E_{i j}^{-1}$ for the undisplaced sensor geometry. Motion of the receivers in the complicated field pattern in the caustic

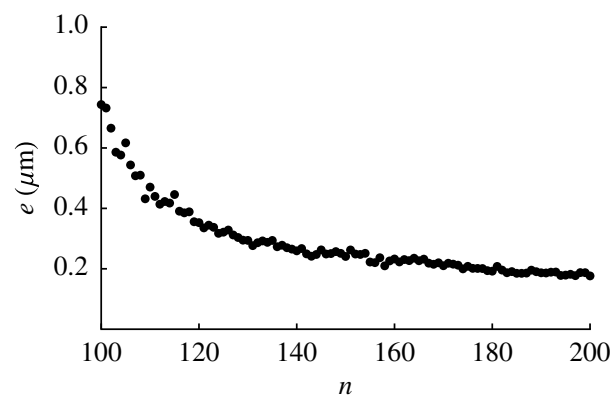

Fig. 4. Measurement error versus number of receivers for optimal, but not circularly symmetric, receiver placement. $(1 \mu \mathrm{m}$ surface error $=0.004 \mathrm{rad}$ phase error at $\lambda=3 \mathrm{~mm}$.) 


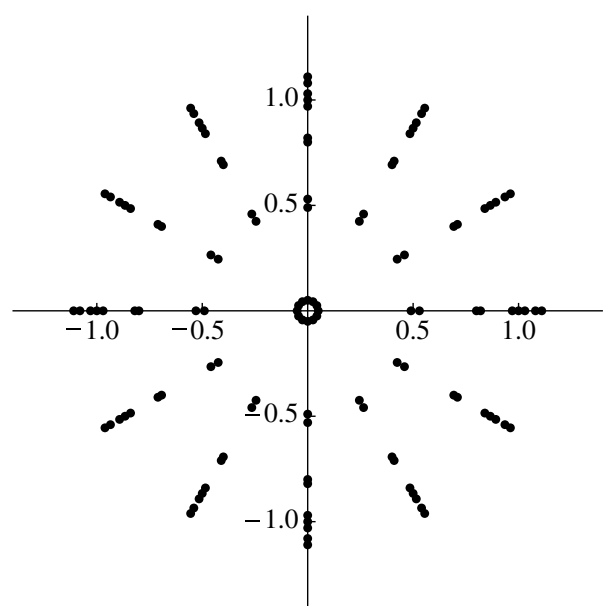

Fig. 5. Optimum circularly symmetric placement for 120 receivers. Receiver positions are in meters.

leads to large, low-order errors in the measured surface, mostly translation, tilt, and defocus. Translation of the primary has no effect on astronomical observations. In addition, we will not use erroneous pointing and focus corrections from a CoC measurement to correct the primary because we can measure the actual pointing and focus errors using astronomical sources. We include this filtering of low-order terms in the simulation by removing translation, tilt, and defocus from both $x_{j}$ and $x_{j}^{\prime}$ just before calculating the measurement error.

Figure 7 shows the measurement error versus sensor decenter, tilt, and despace, and Fig. $\underline{8}$ shows examples of the measured-actual surface profile. The measurement error scales linearly with sensor position error. For a despace, the measurement error has the expected circular symmetry. For a decenter, or a tilt, the pattern of surface errors reflects the motion of the sensor if the receiver configuration is circularly symmetric. For a receiver configuration that is not circularly symmetric, the pattern of surface errors seems to be more dependent on the receiver placement than on the orientation of the sensor position error. $<1 \mu \mathrm{m}$ rms measurement error requires decenter $<20 \mu \mathrm{m}$, tilt $<60 \mu \mathrm{m}$ across the sensor diameter, and despace $<200 \mu \mathrm{m}$. These are fairly stringent requirements, but well within the measurement capabilities of a laser tracker. For closed-loop control
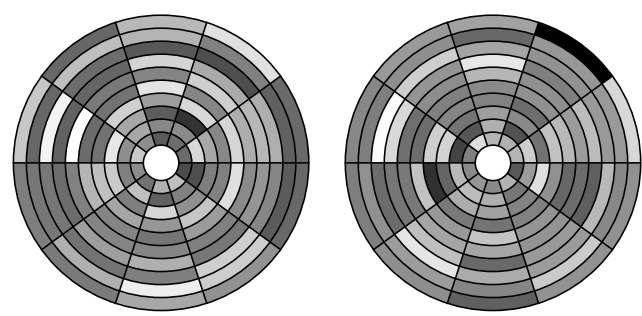

Fig. 6. Actual surface profile (left, gray scale range $\pm 33 \mu \mathrm{m}$ ) and measured-actual profile (right, gray scale range $\pm 0.35 \mu \mathrm{m}$ ) for a simulated $\mathrm{CoC}$ measurement with 120 receivers in the circularly symmetric configuration of Fig. $\underline{5}$.

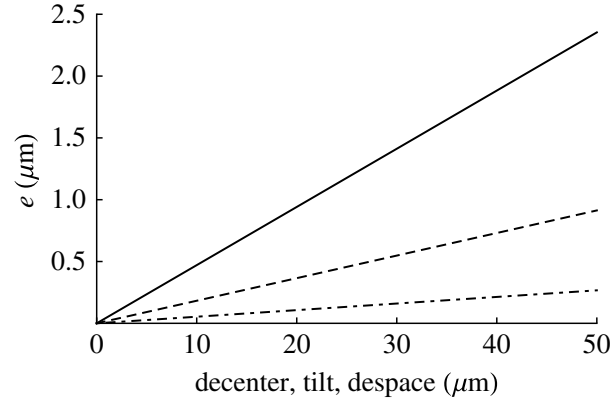

Fig. 7. Measurement error versus sensor decenter (solid line), tilt across the sensor diameter (dashed line), and despace (dash-dotted line).

of the primary, $E_{i j}^{-1}$ could be precomputed for the range of possible sensor positions, so that real-time calculations would involve just the simple matrix multiplication of Eq. (10).

Receiver position errors within the array cause errors in the measured surface profile. For the receiver configuration of Fig. 3, $10 \mu \mathrm{m}$ rms random position errors (e.g., due to manufacturing tolerances) give a surface error of $1 \mu \mathrm{m} \mathrm{rms}$. A $15 \mu \mathrm{m}$ change in sensor radius due to thermal expansion also causes $1 \mu \mathrm{m}$ rms surface error. The change in radius is $\Delta \rho=$ $\rho \alpha \Delta T$, where $\rho$ is the sensor radius, $\alpha$ is the coefficient of thermal expansion of the sensor, and $\Delta T$ is the temperature change. If the sensor feedhorns are mounted on a CFRP plate with $\alpha=10^{-6} \mathrm{~K}^{-1}$ and $\rho=1.5 \mathrm{~m}, \Delta \rho<15 \mu \mathrm{m}$ requires $\Delta T<10 \mathrm{~K}$, so an exposed sensor will need coarse, active temperature control. The $\sim 10 \mu \mathrm{m}$ tolerance on receiver position errors also applies to receiver phase errors and gravitational deformation of the sensor. Phase errors are a concern because the local oscillators for the mixers will probably be distributed through coaxial cables, which will have to be in a temperature controlled environment. An alternative approach would be to couple the local oscillator optically from a source mounted on the back of the secondary.

\section{Obscuration by the Secondary}

The secondary mirror causes two problems. First, it obscures the source, so the inner part of the primary is not illuminated. For CCAT, a ray from the center of
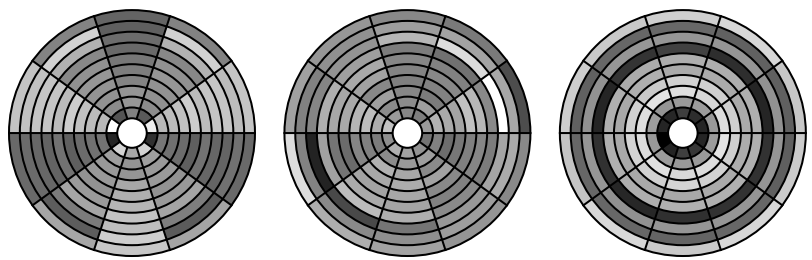

Fig. 8. Measured-actual surface profile for a $15 \mu \mathrm{m}$ decenter (left, gray scale range $\pm 2.0 \mu \mathrm{m}$, decenter towards the bottom of the page), a $17.5 \mu \mathrm{m}$ tilt across the sensor (center, gray scale range $\pm 1.0 \mu \mathrm{m}$, tilt into the page at top right with tilt axis $60^{\circ}$ clockwise from horizontal), and an $80 \mu \mathrm{m}$ despace (right, gray scale range $\pm 0.75 \mu \mathrm{m}$ ). The receiver configuration is circularly symmetric. 


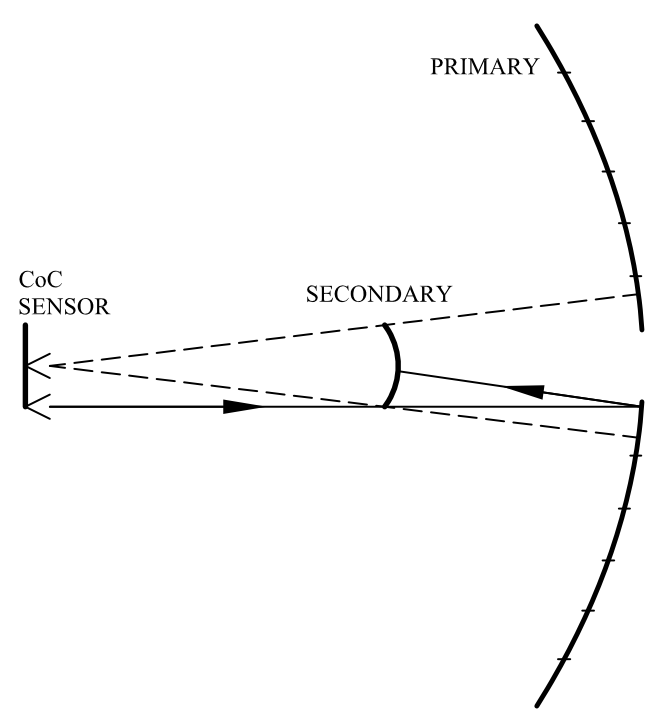

Fig. 9. Obscuration by the secondary. The dashed lines show the shadow of the secondary for a source at the center of the sensor. Moving the source to the edge of the sensor gives access to the inner part of the primary, but some of the reflected rays are obscured by the secondary. The ticks on the primary show the edges of the segments. The vertex of the $3 \mathrm{~m}$ diameter secondary is $9 \mathrm{~m}$ from the vertex of the primary and the sensor is $22.7 \mathrm{~m}$ from the vertex of the primary.

the sensor to the edge of the secondary intercepts the primary at a radius of $2.56 \mathrm{~m}$, which is near the outer edge of the first ring of segments (see Fig. 9). The obscuration problem can be addressed using additional source and receiver positions around the edge of the sensor in order to see around the secondary. The sensor can be as large as the secondary, which is the same size as the central hole in the primary, so a source at the edge of the sensor can see part of the inner edge of the primary. A few sources are needed around the edge of the sensor to sequentially measure patches around the inner part of the primary. The measurements must then be stitched together to form a complete map of the primary. The reflected field returning to the sensor from the inner panels is also obscured by the secondary. This reduces the power at the receiver array by a factor $\sim 10$, so we need $10 \times$ higher source power to measure the inner panels quickly.

The second problem is diffraction at the secondary. This affects both the field incident on the primary and the reflected field returning to the sensor. Ignoring diffraction leads to large measurement errors near the boundary between the illuminated and shadow regions. The problem is illustrated in Fig. 10, which shows the change in phase of the field incident on the primary due to diffraction at the secondary. The peak phase error in Fig. 10 corresponds to a surface profile error of $\sim 0.5 \mu \mathrm{m} \times[12.5 / R(\mathrm{~m})]^{2}$, where $R$ is the radius on the primary. To avoid this systematic error, we must include diffraction when calculating $E_{i j}$, or measure the error, e.g., by comparing a CoC measurement with a shearing interferometer measurement, and apply a correction to $v_{j}^{\prime}$.

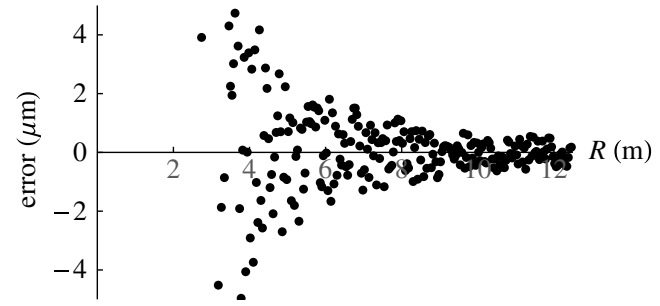

Fig. 10. Surface error corresponding to the change in phase of the field at the primary due to diffraction at the secondary. $R$ is the radius on the primary. The source is at the center of the sensor. The inner region with no points is obscured by the secondary.

\section{Conclusions}

We have demonstrated, by calculation, that the surface profile of a fast paraboloid can be measured at the micrometer level using a millimeter-wave source at the $\mathrm{CoC}$ and an array of receivers to measure the field in the caustic. The source and receivers could be inexpensive, commercially-available, millimeter-wave parts. The $\mathrm{CoC}$ sensor could be mounted on the telescope enclosure, so it would move with the telescope and always be available for slow, closed-loop control of the surface.

The CoC sensor must have at least as many receivers as patches to be measured on the surface. The position of the sensor must be measured to $\sim 20 \mu \mathrm{m}$, which can be done using a laser tracker. A single measurement of the surface must be made quickly enough $(<100 \mu \mathrm{s})$ to freeze sensor motion due to wind buffeting. This requires a source power of order $1 \mathrm{~mW}$ if the receivers are room-temperature mixers. Measurements can be stacked to improve the signalto-noise ratio. The portion of the surface that is obscured by the secondary can be measured by switching the source to a feedhorn at the edge of the array of receivers. Diffraction at the secondary causes errors of a few $\mu \mathrm{m} \mathrm{rms}$ in the recovered surface profile, so a correction must be applied for this effect.

This work was supported by the John B. and Nelly Kilroy Foundation.

\section{References}

1. S. J. E. Radford, R. Giovanelli, T. A. Sebring, and J. Zmuidzinas, "CCAT," Astron. Soc. Pac. Conf. Ser. 417, 113-123 (2009).

2. G. L. Stacey, S. R. Golwala, C. M. Bradford, C. D. Dowell, G. Cortes-Medellin, T. Nikola, J. Zmuidzinas, T. L. Herter, S. J. Radford, J. P. Lloyd, A. W. Blain, R. L. Brown, D. B. Campbell, R. Giovanelli, P. Goldsmith, P. M. Harvey, C. Henderson, W. D. Langer, T. G. Phillips, A. C. S. Readhead, and D. P. Woody, "Instrumentation for the CCAT telescope," Proc. Soc. Photo-Opt. Instrum. Eng. 6275, 62751G (2006).

3. D. P. Woody, S. Padin, and T. Sebring, "CFRP truss for the CCAT $25 \mathrm{~m}$ diameter submillimeter-wave telescope," Proc. Soc. Photo-Opt. Instrum. Eng. 7733, 77332B (2010).

4. J. E. Nelson, T. S. Mast, and S. M. Faber, "The design of the Keck Observatory and Telescope," Keck Observatory Rep. No. 90 (W. M. Keck Observatory, Kamuela, Hawaii, 1985).

5. J. M. Payne, "An optical distance measuring instrument," Rev. Sci. Instrum. 44, 304-306 (1973).

6. J. W. Findlay and J. M. Payne, "An instrument for measuring deformations in large structures," IEEE Trans. Instrum. Meas. 23, 221-226 (1974). 
7. B. Nikolic, R. E. Hills, and J. S. Richer, "Measurement of antenna surfaces from in- and out-of-focus beam maps using astronomical sources," Astron. Astrophys. 465, 679-683 (2007).

8. B. Nikolic, R. M. Prestage, D. S. Balser, C. J. Chandler, and R. E. Hills, "Out-of-focus holography at the Green Bank Telescope," Astron. Astrophys. 465, 685-693 (2007).

9. E. Serabyn, T. G. Phillips, and C. R. Masson, "Surface figure measurements of radio telescopes with a shearing interferometer," Appl. Opt. 30, 1227-1241 (1991).

10. D. Malacara, Optical Shop Testing (Wiley, 1992).

11. D. P. Woody, E. Serabyn, and A. Shinkel, "Measurement, modeling, and adjustment of the $10.4 \mathrm{~m}$ diameter Leighton telescopes," Proc. Soc. Photo-Opt. Instrum. Eng. 3357, 474-485 (1998).

12. E. Serabyn and J. K. Wallace, "Submillimeter pupil-plane wavefront sensing," Proc. Soc. Photo-Opt. Instrum. Eng. 7741, 77410U (2010).

13. E. Serabyn, "Fundamental limits to wavefront sensing in the submillimeter," Proc. Soc. Photo-Opt. Instrum. Eng. 6275, 62750Z (2006).

14. V. L. Krabbendam, T. A. Sebring, F. B. Ray, and J. R. Fowler, "Development and performance of Hobby Eberly Telescope 11 meter segmented mirror," Proc. Soc. Photo-Opt. Instrum. Eng. 3352, 436-445 (1998).
15. J. C. Bennett, A. P. Anderson, P. A. McInnes, and A. J. T. Whittaker, "Microwave holographic metrology of large reflector antennas," IEEE Trans. Antennas Propag. 24, 295-303 (1976).

16. P. F. Scott and M. Ryle, "A rapid method for measuring the figure of a radio telescope reflector," Mon. Not. R. Astron. Soc. 178, 539-545 (1977).

17. S. Padin, Z. Staniszewski, R. Keisler, M. Joy, A. A. Stark, P. A. R. Ade, K. A. Aird, B. A. Benson, L. E. Bleem, J. E. Carlstrom, C. L. Chang, T. M. Crawford, A. T. Crites, M. A. Dobbs, N. W. Halverson, S. Heimsath, R. E. Hills, W. L. Holzapfel, C. Lawrie, A. T. Lee, E. M. Leitch, J. Leong, W. Lu, M. Lueker, J. J. McMahon, S. S. Meyer, J. J. Mohr, T. E. Montroy, T. Plagge, C. Pryke, J. E. Ruhl, K. K. Schaffer, E. Shirokoff, H. G. Spieler, and J. D. Vieira, "South Pole Telescope Optics," Appl. Opt. 47, 4418-4428 (2008).

18. R. H. Dicke, "The measurement of thermal radiation at microwave frequencies," Rev. Sci. Instrum. 17, 268-275 (1946).

19. J. D. Kraus, Radio Astronomy (McGraw-Hill, 1966).

20. P. J. B. Clarricoats and A. D. Oliver, Corrugated Horns for Microwave Antennas (Peter Peregrinus, 1984).

21. J. D. Jackson, Classical Electrodynamics (Wiley, 1998), pp. $483-484$. 